\title{
INEQUALITIES INVOLVING CYLINDRICAL FUNCTIONS OF NEARLY EQUAL ARGUMENT AND ORDER
}

\author{
K. M. SIEGEL AND F. B. SLEATOR
}

Most known bounds on special functions of large argument and order are without limit as the argument approaches the order. In a previous paper [1] a new bound was obtained for the Bessel function of large order in the range where the argument is less than but nearly equal to the order. In this paper finite bounds are obtained for the range of argument greater than but approaching the order. Bounds of the same type are found for the Neumann function.

In [1], there was obtained the following inequality, valid for $x<1$ :

$$
J_{\nu}(\nu x) \leqq \frac{\exp \left(\nu\left(1-x^{2}\right)^{1 / 2}\right) x^{\nu}}{\left(1+\left(1-x^{2}\right)^{1 / 2}\right)^{\nu}} .
$$

Also in Watson [2, p. 255], we find the inequality

$$
\begin{aligned}
\frac{\partial J_{\nu}(\nu x)}{\partial(\nu x)} & \equiv J_{\nu}^{\prime}(\nu x) \\
& \leqq \frac{\left(1+x^{2}\right)^{1 / 4}}{x(2 \pi \nu)^{1 / 2}}\left(\frac{x}{1+\left(1-x^{2}\right)^{1 / 2}}\right)^{\nu} \exp \left(\nu\left(1-x^{2}\right)^{1 / 2}\right)
\end{aligned}
$$

To obtain corresponding inequalities for the range of $x$ greater than but nearly equal to 1 , one need only employ (1), (2), and the standard recursion formulas. Suppose, for example, that $1-1 / \nu<x<1$, and write

$$
J_{\nu-1}(\nu x)=\frac{1}{x} J_{\nu}(\nu x)+J_{\nu}^{\prime}(\nu x) .
$$

The left side is a Bessel function with argument slightly greater than its order. Substitution of (1) and (2) yields

$$
J_{\nu-1}(\nu x) \leqq \frac{\exp \left(\nu\left(1-x^{2}\right)^{1 / 2}\right)}{x}\left(\frac{x}{1+\left(1-x^{2}\right)^{1 / 2}}\right)^{\nu}\left(1+\frac{\left(1+x^{2}\right)^{1 / 4}}{(2 \pi \nu)^{1 / 2}}\right) .
$$

For large $\nu$, the right-hand side here approximates unity as $x \rightarrow 1$, just as in (1). To obtain bounds which are valid over an extended range of $x$, the recursion formulas may be iterated. Thus in the range

$$
\nu<\nu+n-1<\nu x<\nu+n
$$

Received by the editors September 24, 1953. 
we may use inequalities (1) and (2) in the recursion formula involving

$$
J_{\nu-n}(\nu x), \quad J_{\nu}(\nu x), \quad \text { and } J_{\nu}^{\prime}(\nu x),
$$

which is easily obtained by iteration of the standard forms. For example, let $\nu+1<\nu x<\nu+2$. Substituting (1) and (2) into

$$
J_{\nu-2}(\nu x)=\left(\frac{2(\nu-1)}{x^{2} \nu}-1\right) J_{\nu}(\nu x)+\frac{2(\nu-1)}{\nu x} J_{\nu}^{\prime}(\nu x)
$$

we obtain

$$
\begin{aligned}
J_{\nu-2}(\nu x) \leqq & \exp \left(\nu\left(1-x^{2}\right)^{1 / 2}\right)\left(\frac{x}{1+\left(1-x^{2}\right)^{1 / 2}}\right)^{\nu} \\
\cdot & {\left[\frac{2\left(1+x^{2}\right)^{1 / 4}(1-1 / \nu)}{x^{2}(2 \pi \nu)^{1 / 2}}+\frac{2}{x^{2}}\left(1-\frac{1}{\nu}\right)-1\right] . }
\end{aligned}
$$

A shift of index then yields the desired expression.

To obtain a bound on the Neumann function we can utilize the fact that for fixed positive $\nu$, the quantity $(\nu x)\left(J_{\nu}^{2}(\nu x)+N_{\nu}^{2}(\nu x)\right)$ is a monotonically decreasing function of $x$ [3, p. 25]. From this it follows that for $x>1$,

$$
(\nu x)\left[J_{\nu}^{2}(\nu x)+N_{\nu}^{2}(\nu x)\right]<\nu\left[J_{\nu}^{2}(\nu)+N_{\nu}^{2}(\nu)\right]
$$

or

$$
N_{\nu}^{2}(\nu x)<(1 / x)\left[J_{\nu}^{2}(\nu)+N_{\nu}^{2}(\nu)\right]-J_{\nu}^{2}(\nu x) .
$$

Since the last term on the right is negative, we may neglect it and write

$$
N_{\nu}^{2}(\nu x)<(1 / x)\left[J_{\nu}^{2}(\nu)+N_{\nu}^{2}(\nu)\right] .
$$

An upper bound on the first term in the bracket is given by the Cauchy formula $[2$, p. 259]

$$
J_{\nu}(\nu)<\frac{\Gamma(1 / 3)}{2^{2 / 3} 3^{1 / 6} \pi \nu^{1 / 3}} .
$$

A similar upper bound on $N_{\nu}(\nu)$ can be obtained in the following manner.

We have, for nonintegral $\nu$, the following expression:

$$
N_{\nu}(z)=\frac{1}{\sin \nu \pi}\left[\cos \nu \pi J_{\nu}(z)-J_{-\nu}(z)\right] .
$$

Also in $[4$, p. 362] we find the formula 


$$
\begin{aligned}
J_{-\nu}(z)= & \frac{1}{\pi} \int_{0}^{\pi} \cos (z \sin \theta+\nu \theta) d \theta \\
& +\frac{\sin \nu \pi}{\pi} \int_{0}^{\infty} \exp (\nu \theta-z \sinh \theta) d \theta .
\end{aligned}
$$

Putting $z=\nu$, we have

$$
J_{-\nu}(\nu)=\frac{1}{\pi} \int_{0}^{\pi} \cos [\nu(\sin \theta+\theta)] d \theta+\frac{\sin \nu \pi}{\pi} \int_{0}^{\infty} \exp (\nu(\theta-\sinh \theta)) d \theta .
$$

Substitution of a series expansion for $\sinh \theta$ in the second integrand yields

$$
\exp (\nu(\theta-\sinh \theta))=\exp \left(-\frac{\nu \theta^{3}}{3 !}-\frac{\nu \theta^{5}}{5 !}-\cdots\right) \leqq \exp \left(-\frac{\nu \theta^{3}}{3 !}\right),
$$

from which

$$
\begin{aligned}
J_{-\nu}(\nu) \leqq & \frac{1}{\pi} \int_{0}^{\pi}|\cos [\nu(\sin \theta+\theta)]| d \theta \\
& +\frac{|\sin \nu \pi|}{\pi} \int_{0}^{\infty} \exp \left(-\frac{\nu \theta^{3}}{3 !}\right) d \theta \\
\leqq & \frac{1}{\pi} \int_{0}^{\pi}|\cos [\nu(\sin \theta+\theta)]| d \theta+\frac{|\sin \nu \pi|}{\pi} \frac{2^{1 / 3} \Gamma(1 / 3)}{3^{2 / 3} \nu^{1 / 3}} \\
\leqq & \frac{1}{\pi} \int_{0}^{\pi} d \theta+\frac{2^{1 / 3} \Gamma(1 / 3)|\sin \nu \pi|}{3^{2 / 3} \nu^{1 / 3} \pi}=1+\frac{2^{1 / 3} \Gamma(1 / 3)|\sin \nu \pi|}{3^{2 / 3} \nu^{1 / 3} \pi} .
\end{aligned}
$$

Putting this into (4), we get

$$
\begin{aligned}
\left|N_{\nu}(\nu)\right| & \leqq|\cot \nu \pi| J_{\nu}(\nu)+\frac{1}{|\sin \nu \pi|}+\frac{2^{1 / 3} \Gamma(1 / 3)}{3^{2 / 3} \pi \nu^{1 / 3}} \\
& \leqq|\cot \nu \pi| \frac{\Gamma(1 / 3)}{2^{2 / 3} 3^{1 / 6} \pi \nu^{1 / 3}}+\frac{1}{|\sin \nu \pi|}+\frac{2^{1 / 3} \Gamma(1 / 3)}{3^{2 / 3} \pi \nu^{1 / 3}} .
\end{aligned}
$$

Since $N_{\nu}^{2}(\nu x) \leqq(1 / x)\left[J_{\nu}^{2}(\nu)+N_{\nu}^{2}(\nu)\right]$, it follows that

$$
\begin{aligned}
\left|N_{\nu}(\nu x)\right| & \leqq \frac{1}{x^{1 / 2}}\left[\left|J_{\nu}(\nu)\right|+\left|N_{\nu}(\nu)\right|\right] \\
& \leqq \frac{1}{x^{1 / 2}}\left[\left(\frac{1+|\cot \nu \pi|}{2^{2 / 3} 3^{1 / 6}}+\frac{2^{1 / 3}}{3^{2 / 3}}\right) \frac{\Gamma(1 / 3)}{\pi \nu^{1 / 3}}+\frac{1}{|\sin \nu \pi|}\right]
\end{aligned}
$$

or 


$$
\left|N_{\nu}(\nu x)\right| \leqq \frac{1}{x^{1 / 2}}\left[\frac{\Gamma(1 / 3)}{\pi \nu^{1 / 3}}\left(\frac{1+2 / 3^{1 / 2}+|\cot \nu \pi|}{2^{2 / 3} 3^{1 / 6}}\right)+\frac{1}{|\sin \nu \pi|}\right] .
$$

The above bound on $\left|N_{\nu}(\nu x)\right|$ becomes infinite as $\nu$ approaches integral values, for which values, of course, formula (4) is indeterminate. The case of integral $\nu$ can be treated by means of an expression for $N_{n}(n)$ which corresponds to $(5)$. [3, p. 27] yields the formula

$$
\begin{aligned}
N_{n}(n)= & \frac{1}{\pi} \int_{0}^{\pi} \sin [n(\sin \theta-\theta)] d \theta \\
& -\frac{\pi}{2} \exp \left(-i n \frac{\pi}{2}\right) \int_{0}^{\infty} \exp (-n \sinh \theta) \cosh n\left(\theta+\frac{i \pi}{2}\right) d \theta .
\end{aligned}
$$

Here we note that

$$
\exp (-i n \pi / 2) \cosh n(\theta+i \pi / 2)=(1 / 2)\left(e^{n \theta} \pm e^{-n \theta}\right),
$$

the sign depending on the value of $n$. Putting this into (8) we get

$$
\begin{aligned}
N_{n}(n)= & \frac{1}{\pi} \int_{0}^{\pi} \sin [n(\sin \theta-\theta)] d \theta \\
& -\frac{1}{\pi} \int_{0}^{\infty} \exp (-n \sinh \theta)\left(e^{n \theta} \pm e^{-n \theta}\right) d \theta,
\end{aligned}
$$

which yields immediately the inequality

$$
\begin{aligned}
\left|N_{n}(n)\right| \leqq & \frac{1}{\pi} \int_{0}^{\pi}|\sin [n(\sin \theta-\theta)]| d \theta \\
& +\frac{1}{\pi} \int_{0}^{\infty} \exp (-n(\theta+\sinh \theta)) d \theta \\
& +\frac{1}{\pi} \int_{0}^{\infty} \exp (-n(\sinh \theta-\theta)) d \theta .
\end{aligned}
$$

Using the same arguments as before, we obtain

$$
\begin{aligned}
\left|N_{n}(n)\right| & \leqq 1+\frac{1}{\pi} \int_{0}^{\infty} \exp (-2 n \theta) d \theta+\frac{1}{\pi} \int_{0}^{\infty} \exp \left(-\frac{n \theta^{3}}{3 !}\right) d \theta \\
& =1+\frac{1}{2 n \pi}+\frac{2^{1 / 3} \Gamma(1 / 3)}{3^{2 / 3} n^{1 / 3} \pi}
\end{aligned}
$$

from which 


$$
\begin{aligned}
\left|N_{n}(n x)\right| & \leqq \frac{1}{x^{1 / 2}}\left[\frac{\Gamma(1 / 3)}{\pi n^{1 / 3}}\left(\frac{1}{2^{2 / 3} 3^{1 / 6}}+\frac{2^{1 / 3}}{3^{2 / 3}}\right)+\frac{1}{2 n \pi}+1\right] \\
& \leqq \frac{1}{x^{1 / 2}}\left[\frac{.964}{n^{1 / 3}}+\frac{1}{2 n \pi}+1\right] .
\end{aligned}
$$

In specific problems, the condition may arise that $\nu$ is nearly but not exactly an integer, in which case neither (7) nor (9) may be of any use. This case can be treated by means of the following considerations.

On the basis of (4) and (5) we can write

$$
N_{\nu}(\nu)=A+B
$$

where

(11) $A=-\frac{1}{\pi} \int_{0}^{\infty}[\exp (\nu(\theta-\sinh \theta))+\cos \nu \pi \exp (-\nu(\theta+\sinh \theta))] d \theta$,

$$
\begin{aligned}
B= & \frac{\cos \nu \pi}{\pi \sin \nu \pi} \int_{0}^{\pi} \cos [\nu(\sin \theta-\theta)] d \theta \\
& -\frac{1}{\pi \sin \nu \pi} \int_{0}^{\pi} \cos [\nu(\sin \theta+\theta)] d \theta .
\end{aligned}
$$

The customary substitution in (11) yields at once

$$
|A|<\frac{1}{\pi} \mid \int_{0}^{\infty}\left[\exp \left(-\frac{\nu \theta^{3}}{3 !}\right)+|\cos \nu \pi| \exp (-2 \nu \theta)\right] d \theta
$$

from which

$$
|A|<\frac{1}{\pi}\left[\frac{2^{1 / 3} \Gamma(1 / 3)}{3^{2 / 3} \nu^{1 / 3}}+\frac{|\cos \nu \pi|}{2 \nu}\right] .
$$

In considering $B$, we must examine two separate cases:

(1) $\nu=2 n+\epsilon$,

(2) $\nu=2 n+1+\epsilon$,

$$
n=0,1,2, \cdots ; 1 \gg \epsilon>0 \text {. }
$$

For case (1), we rewrite (12) in the form

$$
\begin{aligned}
B= & \frac{\cos \nu \pi-1}{\pi \sin \nu \pi} \int_{0}^{\pi} \cos [\nu(\sin \theta-\theta)] d \theta \\
& +\frac{1}{\pi \sin \nu \pi} \int_{0}^{\pi}\{\cos [\nu(\sin \theta-\theta)]-\cos [\nu(\sin \theta+\theta)]\} d \theta .
\end{aligned}
$$


Using the identity

$$
\cos (C-D)-\cos (C+D)=2(\sin C)(\sin D)
$$

in the second integral, we obtain

$$
\begin{aligned}
B= & \frac{\cos \nu \pi-1}{\pi \sin \nu \pi} \int_{0}^{\pi} \cos [\nu(\sin \theta-\theta)] d \theta \\
& +\frac{2}{\pi \sin \nu \pi} \int_{0}^{\pi} \sin (\nu \sin \theta) \sin \nu \theta d \theta .
\end{aligned}
$$

Now set $\nu=2 n+\epsilon$ and in the second integral substitute $\theta=\phi+\pi / 2$. Then

$$
\begin{aligned}
B= & \frac{\cos \epsilon \pi-1}{\pi \sin \epsilon \pi} \int_{0}^{\pi} \cos [(2 n+\epsilon)(\sin \theta-\theta)] d \theta \\
& +\frac{2 \cos \epsilon \pi / 2}{\pi \sin \epsilon \pi} \int_{-\pi / 2}^{\pi / 2} \sin (\nu \cos \phi) \sin \nu \phi d \phi \\
+ & \frac{2 \sin \epsilon \pi / 2}{\pi \sin \epsilon \pi} \int_{-\pi / 2}^{\pi / 2} \sin [(2 n+\epsilon) \cos \phi] \cos [(2 n+\epsilon) \phi] d \phi .
\end{aligned}
$$

Here the second integral vanishes because the integrand is an odd function and the limits are symmetrical. Since the integrands in the remaining terms are always equal to or less than 1 , we have the inequality

$$
|B| \leqq\left|\frac{\cos \epsilon \pi-1}{\pi \sin \epsilon \pi}\right| \cdot \pi+\left|\frac{2 \sin \epsilon \pi / 2}{\pi \sin \epsilon \pi}\right| \cdot \pi .
$$

Substituting the series expansions for sin and cos, and then replacing the quotients by their upper bounds, we obtain

$$
|B| \leqq\left|\frac{(\epsilon \pi)^{2} / 2}{\epsilon \pi-(\epsilon \pi)^{3} / 6}\right|+\left|\frac{\epsilon \pi}{\epsilon \pi-(\epsilon \pi)^{3} / 6}\right|
$$

or, since $1 /(1-x)<1+2 x$ for $0<x<1 / 2$,

$$
|B| \leqq \frac{\epsilon \pi}{2}\left(1+\frac{(\epsilon \pi)^{2}}{3}\right)+1+\frac{(\epsilon \pi)^{2}}{3}=1+\frac{\epsilon \pi}{2}+\frac{(\epsilon \pi)^{2}}{3}+\frac{(\epsilon \pi)^{3}}{6}
$$

$$
|B| \leqq 1+\epsilon \pi+\frac{(\epsilon \pi)^{2}}{2 !}+\frac{(\epsilon \pi)^{3}}{3 !} \cong e^{\epsilon \pi}
$$

Putting (13) and (14) into (10) we obtain the following bound on $\left|N_{\nu}(\nu)\right|$, which remains finite as $\epsilon \rightarrow 0$, 


$$
\left|N_{\nu}(\nu)\right|<\frac{1}{\pi}\left[\frac{2^{1 / 3} \Gamma(1 / 3)}{3^{2 / 3} \nu^{1 / 3}}+\frac{|\cos \nu \pi|}{2 \nu}\right]+e^{\epsilon \pi} .
$$

For case (2) $(\nu=2 n+1+\epsilon)$ we rewrite (12) in a different form,

$$
\begin{aligned}
B= & \frac{\cos \nu \pi+1}{\pi \sin \nu \pi} \int_{0}^{\pi} \cos [\nu(\sin \theta-\theta)] d \theta \\
& -\frac{1}{\pi \sin \nu \pi} \int_{0}^{\pi}\{\cos [\nu(\sin \theta-\theta)]+\cos [\nu(\sin \theta+\theta)]\} d \theta
\end{aligned}
$$

and in the second integral use the identity

$$
\cos (C-D)+\cos (C+D)=2(\cos C)(\cos D)
$$

and the substitution $\theta=\phi+\pi / 2$ to obtain

$$
\begin{aligned}
B= & \frac{\cos \nu \pi+1}{\pi \sin \nu \pi} \int_{0}^{\pi} \cos [\nu(\sin \theta-\theta)] d \theta \\
& -\frac{2 \cos \nu \pi / 2}{\pi \sin \nu \pi} \int_{-\pi / 2}^{\pi / 2} \cos (\nu \cos \phi) \cos \nu \phi d \phi \\
& +\frac{2 \sin \nu \pi / 2}{\pi \sin \nu \pi} \int_{-\pi / 2}^{\pi / 2} \cos (\nu \cos \phi) \sin \nu \phi d \phi .
\end{aligned}
$$

Here the third integral vanishes due to symmetry, and by our earlier reasoning,

$$
|B| \leqq\left|\frac{\cos \nu \pi+1}{\sin \nu \pi}\right|+\left|\frac{2 \cos \nu \pi / 2}{\sin \nu \pi}\right| .
$$

Putting $\nu=2 n+1+\epsilon$ and proceeding as before, we have successively

$$
\begin{aligned}
|B| & \leqq\left|\frac{-\cos \epsilon \pi+1}{-\sin \epsilon \pi}\right|+\left|\frac{2 \sin \epsilon \pi / 2}{\sin \epsilon \pi}\right| \\
& \leqq\left|\frac{-1+(\epsilon \pi)^{2} / 2+1}{-\epsilon \pi+(\epsilon \pi)^{3} / 3 !}\right|+\left|\frac{\epsilon \pi}{\epsilon \pi-(\epsilon \pi)^{3} / 3 !}\right| \\
& \leqq \frac{\epsilon \pi}{2}\left(1+\frac{(\epsilon \pi)^{2}}{3}\right)+1+\frac{(\epsilon \pi)^{2}}{3} \\
& \leqq 1+\frac{\epsilon \pi}{2}+\frac{(\epsilon \pi)^{2}}{3}+\frac{(\epsilon \pi)^{3}}{6} \\
& \leqq e^{\epsilon \pi},
\end{aligned}
$$

which demonstrates that the same bound (15) applies whether $\nu$ 
approaches an even or odd integer.

It can be shown in a similar manner that (15) is also an upper bound on $\left|N_{\nu}(\nu)\right|$ in case $-1 / 2<\epsilon<0$.

Substituting (15) into (6) yields the desired bound on $\left|N_{\nu}(\nu x)\right|$,

$$
\left|N_{\nu}(\nu x)\right| \leqq \frac{1}{x^{1 / 2}}\left[\frac{.964}{\nu^{1 / 3}}+\frac{|\cos \nu \pi|}{2 \nu \pi}+e^{\epsilon \pi}\right]
$$

where $\epsilon$ is the difference between $\nu$ and the greatest integer in $\nu$. This reduces to the previously obtained bound (9) when $\nu \rightarrow n$.

\section{REFERENCES}

1. K. M. Siegel, An inequality involving Bessel functions of argument nearly equal to their order, Proc. Amer. Math. Soc. vol. 4 (1953) pp. 858-859.

2. G. N. Watson, A treatise on the theory of Bessel functions, $2 \mathrm{~d}$ ed., Cambridge University Press, 1952.

3. W. Magnus and F. Oberhettinger, Formulas and theorems for the special functions of mathematical physics, Chelsea Publishing Company, 1949.

4. E. T. Whittaker and G. N. Watson, $A$ course of modern analysis, 4 th ed., Cambridge University Press, 1952.

Willow Run Research Center, University of Michigan 